\title{
MAXIMAL OPERATOR ON THE SPACE OF CONTINUOUS FUNCTIONS
}

\author{
Przemysław Górka \\ Warsaw University of Technology, Department of Mathematics and Information Sciences \\ Pl. Politechniki 1, 00-661 Warsaw, Poland; pgorka@mini.pw.edu.pl
}

\begin{abstract}
We study the maximal operator on continuous functions in the setting of metric measure spaces. The boundedness is proven for metric measure spaces satisfying an annular decay property.
\end{abstract}

\section{Introduction}

It is well known that when $1<p \leq \infty$, the Hardy-Littlewood maximal operator $\mathcal{M}$ is bounded on $L^{p}(X, d, \mu)$, where $(X, d, \mu)$ is a doubling metric measure space (see e.g. [4]). Maximal operator has been also studied in different function spaces, e.g., Banach function spaces [6], Sobolev spaces [5], Lebesgue spaces with variable exponent [2], generalized Orlicz spaces [3].

More recently, Buckley proved [1] the following result:

Suppose that $0<t, \delta \leq 1$. If $(X, d, \mu)$ satisfies the $\delta$-annular decay property and $\mu$ is doubling, then $\mathcal{M}: C^{0, t}(X) \rightarrow C^{0, s}(X)$, where $s=\min (t, \delta)$.

On the other hand, if no annular decay property is assumed, then $\mathcal{M} f$ can fail to be continuous, even if $f \in C^{0,1}(X)$ (see [1, Example 1.4]). Nevertheless, we prove the following theorem about continuity of maximal operator on the space of continuous functions $C(X)$.

Theorem A. Suppose that $0<\delta \leq 1$, and that $(X, d, \mu)$ satisfies the $\delta$-annular property. Then $\mathcal{M}: C(X) \rightarrow C(X)$ and the following estimate holds

$$
\|\mathcal{M} f\|_{C(X)} \leq\|f\|_{C(X)} \text {. }
$$

The remainder of the paper is structured as follows. In Section 2, we introduce the notations and recall the definitions. The proof of Theorem A is contained in the last section.

\section{Preliminaries}

Let $(X, d, \mu)$ be a metric measure space equipped with a metric $d$ and the Borel measure $\mu$. We assume that the measure of every open nonempty set is positive and that the measure of every bounded set is finite. We say that $\mu$ is doubling if there exists a constant $C_{\mu}>0$ such that $\mu(B(x, 2 r)) \leq C_{\mu} \mu(B(x, r))$ for every ball $B(x, r) .{ }^{1}$ We shall denote the average of integrable function $f$ over the measurable set $A$ in the following manner

$$
f_{A} f d \mu=\frac{1}{\mu(A)} \int_{A} f d \mu .
$$

https://doi.org/10.5186/aasfm.2021.4633

2020 Mathematics Subject Classification: Primary 42B25, 43A85.

Key words: Metric measure spaces, annular decay property, maximal operator.

${ }^{1}$ Constant $C_{\mu}$ is called the doubling constant of measure $\mu$. 
The maximal function $\mathcal{M} f$ of a locally integrable function $f: X \rightarrow \mathbf{R}$ is defined by

$$
\mathcal{M} f(x)=\sup _{r>0} f_{B(x, r)}|f| d \mu .
$$

Let us recall the notion of annular decay property [1]. Given $\delta \in(0,1]$, we say that the space $(X, d, \mu)$ satisfies the $\delta$-annular decay property if there exists a constant $K \geq 1$ such that for all $x \in X, r>0,0<\epsilon<1$, we have

$$
\mu(B(x, r) \backslash B(x, r(1-\epsilon))) \leq K \epsilon^{\delta} \mu(B(x, r)) .
$$

One can easily convince oneself that $\mathbf{R}^{n}$ with the Lebesgue measure satisifies 1-annular decay property. Furthermore, if $(X, d, \mu)$ is a lenght metric measure space with a doubling measure $\mu$, then $X$ has the $\delta$-annular decay property for some $\delta \in$ $(0,1]$ dependent on a doubling constant of $\mu$ (see [1, Corollary 2.2]).

Finally, let $(X, d)$ be a metric space, by $C(X)$ we denote the space of continuous functions on $X$ such that the norm

$$
\|f\|_{C(X)}=\sup _{x \in X}|f(x)|
$$

is finite. Furthermore, for $s \in(0,1]$ we denote by $C^{0, s}(X)$ the Hölder space, i.e. the space of functions $f \in C(X)$ such

$$
\|f\|_{C^{0, s}(X)}:=\|f\|_{C(X)}+\sup _{x \neq y} \frac{|f(x)-f(y)|}{d(x, y)^{s}}<\infty .
$$

\section{Proof of the main result}

We shall start with the following result.

Lemma 3.1. If $f \in L_{\mathrm{loc}}^{1}(X)$, then $\mathcal{M} f$ is lower semicontinuous.

Proof. Let $t \in \mathbf{R}$ and

$$
L_{t}=\{x \in X: \mathcal{M} f(x)>t\} .
$$

We shall prove that $L_{t}$ is open. For this purpose we fix $x \in L_{t}$, then from the very definition of the maximal function, there exists $r$ such that

$$
t<\frac{1}{\mu(B(x, r))} \int_{B(x, r)}|f| d \mu .
$$

Next, since $B(x, r)=\bigcup_{n=1}^{\infty} \bar{B}(x, r-1 / n)$, we have ${ }^{2}$

$$
\int_{B(x, r)}|f| d \mu=\lim _{n \rightarrow \infty} \int_{\bar{B}(x, r-1 / n)}|f| d \mu .
$$

Therefore, for sufficiently large $n$ we have

$$
t<\frac{1}{\mu(B(x, r))} \int_{\bar{B}(x, r-1 / n)}|f| d \mu .
$$

Moreover, let us observe that for $z \in B(x, 1 / 2 n)$ we have

$$
\bar{B}(x, r-1 / n) \subset B(z, r-1 / 2 n) \subset B(x, r) .
$$

Thus, finally, for $z \in B(x, 1 / 2 n)$

$$
t<\frac{1}{\mu(B(z, r-1 / 2 n))} \int_{B(z, r-1 / 2 n)}|f| d \mu \leq \mathcal{M} f(z),
$$

\footnotetext{
${ }^{2}$ The notation $\bar{B}(y, R):=\{z \in X: d(y, z) \leq R\}$ is used for closed balls.
} 
and in this way we have proved that the set $L_{t}$ is indeed open.

Proof of Theorem A. Of course, the estimate is obvious. So, we only need to prove that for $f \in C(X)$, the maximal function $\mathcal{M} f$ is continuous. We can assume that $\|f\|_{C(X)}>0$. By Lemma 3.1 we have that $\mathcal{M} f$ is lower semicontinuous. Therefore, we have to show that $\mathcal{M} f$ is upper semicontinuous. For this purpose we shall prove that for $t \in \mathbf{R}$ the set

$$
U_{t}=\{x \in X: \mathcal{M} f(x)<t\}
$$

is open. Let us fix $x_{0} \in U_{t}$ and let $A:=\mathcal{M} f\left(x_{0}\right)$, then for all $r>0$

$$
f_{B\left(x_{0}, r\right)}|f| d \mu \leq A<t
$$

By continuity of $f$ there exists $\sigma \in(0,1)$ such that

$$
|| f\left(x_{0}\right)|-| f(y)|| \leq \frac{t-A}{4}, \quad \text { for } y \in B\left(x_{0}, \sigma\right) .
$$

Next, let us define

$$
R:=\frac{1}{2} \min \left(\frac{\sigma^{2}}{4},\left(\frac{t-A}{2 K 2^{\delta}\|f\|_{C(X)}}\right)^{2 / \delta}\right),
$$

and we shall prove that $B\left(x_{0}, R\right) \subset U_{t}$. For this purpose we fix $x \in B\left(x_{0}, R\right)$. It is enought to prove that for $r>0$

$$
f_{B(x, r)}|f| d \mu<\frac{t+A}{2} .
$$

We shall consider two cases: $r \geq \sqrt{d\left(x, x_{0}\right)}$ and $r<\sqrt{d\left(x, x_{0}\right)}$.

Case: $r \geq \sqrt{d\left(x, x_{0}\right)}$. Since

$$
B(x, r) \subset B\left(x_{0}, r+d\left(x, x_{0}\right)\right) \subset B\left(x, r+2 d\left(x, x_{0}\right)\right),
$$

we have

$$
\begin{aligned}
& f_{B(x, r)}|f| d \mu=f_{B(x, r)}|f| d \mu-f_{B\left(x_{0}, r+d\left(x, x_{0}\right)\right)}|f| d \mu+f_{B\left(x_{0}, r+d\left(x, x_{0}\right)\right)}|f| d \mu \\
& \leq\left(\frac{1}{\mu(B(x, r))}-\frac{1}{\mu\left(B\left(x_{0}, r+d\left(x, x_{0}\right)\right)\right)}\right) \int_{B(x, r)}|f| d \mu+f_{B\left(x_{0}, r+d\left(x, x_{0}\right)\right)}|f| d \mu \\
& \leq\left(\frac{1}{\mu(B(x, r))}-\frac{1}{\mu\left(B\left(x, r+2 d\left(x, x_{0}\right)\right)\right)}\right) \int_{B(x, r)}|f| d \mu+A \\
& \leq\left(\frac{\mu\left(B\left(x, r+2 d\left(x, x_{0}\right)\right)\right)-\mu(B(x, r))}{\mu\left(B\left(x, r+2 d\left(x, x_{0}\right)\right)\right)}\right)\|f\|_{C(X)}+A .
\end{aligned}
$$

Next, by the $\delta$-annular decay property and assumption $r \geq \sqrt{d\left(x, x_{0}\right)}$ we get

$$
\begin{aligned}
f_{B(x, r)}|f| d \mu & \leq K\left(\frac{2 d\left(x, x_{0}\right)}{r+2 d\left(x, x_{0}\right)}\right)^{\delta}\|f\|_{C(X)}+A \\
& \leq K\left(\frac{2 d\left(x, x_{0}\right)}{\sqrt{d\left(x, x_{0}\right)}+2 d\left(x, x_{0}\right)}\right)^{\delta}\|f\|_{C(X)}+A \\
& \leq K 2^{\delta} d\left(x, x_{0}\right)^{\delta / 2}\|f\|_{C(X)}+A \leq K 2^{\delta} R^{\delta / 2}\|f\|_{C(X)}+A \\
& <(t-A) / 2+A=(t+A) / 2 .
\end{aligned}
$$


Case: $r<\sqrt{d\left(x, x_{0}\right)}$. Let us observe that

$$
B(x, r) \cup B\left(x_{0}, r+d\left(x, x_{0}\right)\right) \subset B\left(x_{0}, \sigma\right) .
$$

Indeed, for $y \in B(x, r)$ we have

$$
\begin{aligned}
d\left(y, x_{0}\right) & \leq d(y, x)+d\left(x, x_{0}\right)<r+d\left(x, x_{0}\right) \\
& <\sqrt{d\left(x, x_{0}\right)}+d\left(x, x_{0}\right)<\sqrt{R}+R \leq 2 \sqrt{R}<\sigma .
\end{aligned}
$$

Moreover, since

$$
\begin{aligned}
r+d\left(x, x_{0}\right) & \leq \sqrt{d\left(x, x_{0}\right)}+d\left(x, x_{0}\right) \\
& <\sqrt{R}+R \leq 2 \sqrt{R}<\sigma,
\end{aligned}
$$

we obtain $B\left(x_{0}, r+d\left(x, x_{0}\right)\right) \subset B\left(x_{0}, \sigma\right)$.

Next, by (1) we get

$$
\begin{aligned}
f_{B(x, r)}|f| d \mu= & f_{B(x, r)}\left(|f|-\left|f\left(x_{0}\right)\right|\right) d \mu-f_{B\left(x_{0}, r+d\left(x, x_{0}\right)\right)}\left(|f|-\left|f\left(x_{0}\right)\right|\right) d \mu \\
& +f_{B\left(x_{0}, r+d\left(x, x_{0}\right)\right)}|f| d \mu \\
\leq & f_{B(x, r)}|| f|-| f\left(x_{0}\right)|| d \mu+f_{B\left(x_{0}, r+d\left(x, x_{0}\right)\right)}|| f|-| f\left(x_{0}\right)|| d \mu+A \\
\leq & (t-A) / 4+(t-A) / 4+A=(t+A) / 2 .
\end{aligned}
$$

This finishes the proof of $(2)$ and hence the whole proof of Theorem A is complete.

Acknowledgements. Thanks to the referee for carefully reading this work.

\section{References}

[1] Buckley, S. M: Is the maximal function of a Lipschitz function continuous? - Ann. Acad. Sci. Fenn. Math. 24:2, 1999, 519-528.

[2] Diening, L.: Maximal function on generalized Lebesgue spaces $L^{p(\cdot)}$. - Math. Inequal. Appl. 7, 2004, 245-253.

[3] Hëstö, P.: The maximal operator on generalized Orlicz spaces. - J. Funct. Anal. 269, 2015, 4038-4048.

[4] Heinonen, J.: Lectures on analysis on metric spaces. - Universitext, 2001.

[5] Kinnunen, J.: The Hardy-Littlewood maximal function of a Sobolev function. - Israel J. Math. 100, 1997, 117-124.

[6] Shimogaki, T.: The Hardy-Littlewood majorants in function spaces. - J. Math. Soc. Japan $17,1965,365-373$.

Received 11 May $2020 \bullet$ Accepted 29 June 2020 